\title{
Lateral Flow Immunoassay for Naked Eye Detection of Mycobacterium tuberculosis
}

\author{
Nazifah Ariffin, ${ }^{1}$ Nor Azah Yusof $\mathbb{D}^{\mathrm{D}},{ }^{1,2}$ Jaafar Abdullah $\mathbb{D}^{1,},{ }^{1,2}$ Siti Fatimah Abd Rahman $\mathbb{D}^{1},{ }^{1}$ \\ Nurul Hanun Ahmad Raston, ${ }^{3}$ Norzila Kusnin, ${ }^{1}$ and Siti Suraiya $\mathbb{B D}^{4}$ \\ ${ }^{1}$ Institute of Advanced Technology, Universiti Putra Malaysia, 43400 Serdang, Selangor, Malaysia \\ ${ }^{2}$ Chemistry Department, Faculty of Science, Universiti Putra Malaysia, 43400 Serdang, Selangor, Malaysia \\ ${ }^{3}$ School of Biosciences and Biotechnology, Faculty of Science and Technology, Universiti Kebangsaan Malaysia, 43600 Bangi, \\ Selangor, Malaysia \\ ${ }^{4}$ Department of Microbiology and Parasitology, Universiti Sains Malaysia, 16150 Kota Bharu, Kelantan, Malaysia
}

Correspondence should be addressed to Nor Azah Yusof; azahy@upm.edu.my

and Siti Fatimah Abd Rahman; siti_fatimah0410@yahoo.com

Received 23 August 2019; Revised 23 November 2019; Accepted 2 December 2019; Published 24 January 2020

Academic Editor: Hana Vaisocherova - Lisalova

Copyright (C) 2020 Nazifah Ariffin et al. This is an open access article distributed under the Creative Commons Attribution License, which permits unrestricted use, distribution, and reproduction in any medium, provided the original work is properly cited.

\begin{abstract}
Tuberculosis (TB) is an infectious disease caused by Mycobacterium tuberculosis. Detection and control of infectious diseases is a major problem, especially in developing countries. Lateral flow immunoassay (LFIA) has been introduced as a handheld immunoassay-based point-of-care platform for an automated detection of TB. The CFP10-ESAT6 antigen of M. tuberculosis was used as the target in early detection of TB using LFIA strip-based POC strategy. An interesting platform based on optical signals is implemented as a colour change in the detection area that is visible to the naked eye. The gold nanoparticles (AuNPs) were used as the colour probe for the detection of a target of interest. The high-resolution transmission electron microscopy (HRTEM) image and ultraviolet-visible spectrophotometer (UV-Vis) analysis confirmed that the synthesized AuNPs were appropriate for the immunoassay designed. The platform consists of AuNPs conjugated with specific antibodies (Ab) to capture the antigen of $M$. tuberculosis. Under the capillary effect, sandwich immunoreactions of AuNP-Ab-antigen were performed on the test pad of the immunostrip, which can be observed by the colour signal on the test line of the strip with a short assay time. Furthermore, the newly developed biosensor was utilized in CFP10-ESAT6 antigen detection in human sputum specimens with satisfactory results. The characteristic coloured bands enable visual detection (naked eye) of target analyte without instrumentation. This noninvasive diagnose system which is sputum-based detection could provide user-friendly and affordable diagnostic tests in developing countries.
\end{abstract}

\section{Introduction}

Tuberculosis (TB) is one of the deadliest infectious diseases that became a significant public health problem worldwide [1]. The disease is mainly caused by the infection of Mycobacterium tuberculosis, which can be transmitted via minute aerosol droplets such as coughing, sneezing, or even talking by an infected TB person [2]. This airborne contagious disease caused more than nine million new cases annually, making $\mathrm{TB}$ the second leading cause of death after human immunodeficiency virus (HIV) infection [3]. The primary reasons for the high prevalence rate of $\mathrm{TB}$ include inadequate access to effective diagnostic methods and inability to treat all infectious cases of pulmonary $\mathrm{TB}$ in a timely fashion, allowing continued $M$. tuberculosis transmission within communities.

The current gold standard for TB diagnosis is sputum smear microscopy, chest radiology, and solid culture $[4,5]$. Even though the methods are capable of diagnosing TB, these tests are limited by poor sensitivity, low specificity, and a time-consuming process [6]. The nucleic acid amplification-based systems have been developed and offer 
relative improvements in sensitivity, specificity, and rapid detection of $M$. tuberculosis over conventional techniques. However, this method requires a high standard of technical competence and high-cost equipment and is used only in proficient laboratories that can afford reference reagents to monitor the assay performance [7-9]. Since TB is a disease of poverty, in which over $90 \%$ of the worldwide burden of TB takes place in low-income and middle-income countries, there is an unmet need for reliable diagnostic methods to identify TB disease rapidly, efficiently, and accurately in an economical way to be used in resourceconstrained settings.

Paper-based immunoassays represent a powerful technique with high relevance in biosensing as it achieved the requirements needed for point-of-care (POC) devices, including rapid detection, a small amount of sample, and low cost so that more people can conveniently receive costeffective healthcare in resource-limited areas [10]. The paper-based POC immunoassays are generally composed of three major components, i.e., paper as the substrate, antibodies as the detection element, and reporter molecules as the signal-transforming element. Lateral flow immunoassay (LFIA), also known as strip-based biosensing, is one of the existing paper-based platforms that represent the most favourable strategy for on-site and one-shot sensor (disposable) analysis $[10,11]$. It is worth mentioning that electrochemical approaches are also taking advantage of lateral flow strips [12]. However, LFIA has some drawbacks; for example, at low concentrations of analyte, this technology may present problems in terms of sensitivity [13].

Incorporation of nanoparticles (NPs), such as gold nanoparticles (AuNPs) with different biorecognition elements (antibodies, peptide arrays, polymers, and aptamers), provides an effective strategy to enhance the performance of the detection systems. The AuNP-based paper biosensor initiated intensive studies recently for the diagnosis of various infectious diseases, such as malaria [14], hepatitis B virus (HBV) [15], dengue [16], Ebola virus (EBOV) [17], and other viruses $[18,19]$. In this context, three types of paper-based immunoassays have been demonstrated, including colorimetric-based, fluorescence-based, and electrochemicalbased immunoassays. Although the fluorescence-based and electrochemical-based immunoassays show advanced merits in terms of sensitivity, they also involved the issues of multistep operation and reader-dependent during detection, which reduces the convenience of end-users. Meanwhile, the advantages of colorimetric-based immunoassay include low cost, equipment-free, rapid, naked-eye readable, and suitable for high-throughput screening on-site; however, the improvements in accuracy, sensitivity, and quantification are still its challenges [10].

Concerning handheld detection platforms for the POC, particularly for TB detection, colorimetric-based LFIA appears to be a good start since this method realised rapid diagnostics in the field conditions as well as simple operation. In addition, early detection is so critical to enabling timely initiation of antituberculosis treatment, which is the key to reduce the mortality as well as to avoid TB epidemic. To achieve that, we have focused on M. tuberculosis secretory proteins suitable for the early detection of TB. The $10 \mathrm{kDa}$ culture filtrate protein (CFP10) and $6 \mathrm{kDa}$ early secreted antigen target (ESAT6) were found to be encoded by the Rv3874 and Rv3875 genes, respectively. These genes are located in the region of difference-1 (RD-1) of the virulent $M$. tuberculosis genome, which are potent T-cell antigens recognised in over $70 \%$ of TB patients $[20,21]$. An exciting discovery was that ESAT6 and its protein partner, CFP10, bind tightly to each other in a ratio of $1: 1$ through hydrophobic and Van der Waals interactions to form an ESAT6-CFP10 protein complex. Both proteins adopt a stable, fully folded structure in the complex, with about two-thirds of the backbone in a regular helical conformation [22]. Meher and coworkers reported that the complex formation of ESAT6-CFP10 resulted in structural changes, enhanced thermodynamics and biochemical stability, and loss of binding to phospholipid membranes [23]. This suggests that ESAT6 and CFP10 are active as a complex and may confer several functional advantages, including tighter control of the activity of regulatory proteins such as transcription factors, and a general mechanism for increasing the specificity of proteinprotein and protein-nucleic acid interactions [22, 23]. Besides, the work reported by Renshaw and coworkers showed that the combination of CFP10-ESAT6 antigen increased the diagnostic performance of assay as compared to a single antigen [24].

Herein in this work, the merits of LFIA and CFP10ESAT6 as the identification protein biomarker were combined, and for the first time, we developed a disposable immunoassay-based test strip as a novel biosensor for rapid detection of TB using the naked eye. Noteworthily, the strategy that incorporated AuNPs as the label plays a crucial role in enhancing the visual effect and the response intensities of LFIA. The design and response principle of this newly developed method are illustrated in Figure 1. Antibodies are bound on different positions of the LFIA to capture target antigens, and the coloured detector reagents labelled (AuNPs) on antibodies give the coloured responses on the test zone and control zone on the LFIA. The characteristic coloured bands enable visual detection (naked eye) of the target analyte. A sandwich immunoassay format was used to amplify the detection signal as well as increase the selectivity of the sensor towards the TBspecific biomarkers. The appearance of both coloured test line and coloured control line indicate a positive result (Figure 1(a)), whereas the observation of a single coloured control line indicates a negative result (Figure 1(b)). The control line indicates that the sample has migrated across the membrane as intended, regardless of whether the analyte is present or not in the sample. If no coloured lines appear at all, it is considered an invalid result; thus, the test must be repeated.

We also demonstrated the effectiveness of developed LFIA for the detection of CFP10-ESAT6 in sputum, collected from human samples of TB-infected persons. The study suggested that the blood test-free method through sputum detection of biomarkers might be of value in the early detection of $\mathrm{TB}$ disease, especially in HIV-positive cases, and thus could provide wide potential applications 


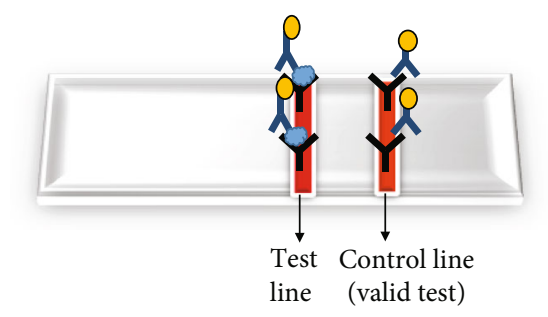

CFP10-ESAT6 (target analyte)

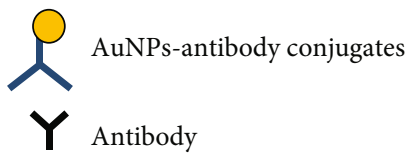

(a)

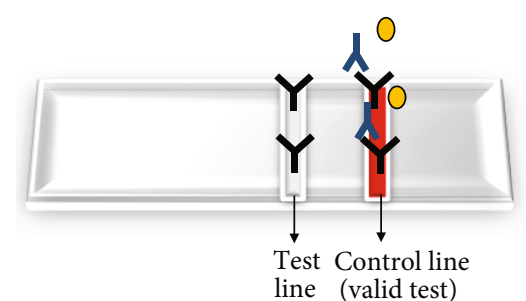

CFP10-ESAT6 (target analyte)

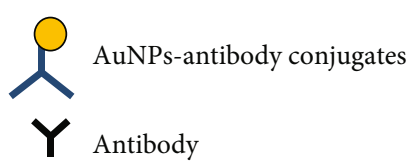

(b)

FIgURE 1: Schematic illustration of the detection principle based on lateral flow test strip biosensor with colloidal gold as label. (a) Positive result indicating $M$. tuberculosis detected by observation of two coloured lines (TB-infected patient). (b) Negative result indicating no presence of M. tuberculosis in sample (healthy person). Only one coloured line appeared.

in clinical analysis and at-home usage without peripheral laboratory implementation.

\section{Materials and Methods}

2.1. Materials and Reagents. Polyclonal anti-CFP10 antibody, rabbit anti $M$. tuberculosis, rabbit anti-M. tuberculosis HRP conjugate, goat anti-rabbit IgG antibody, M. tuberculosis ESAT-6-like protein esxB (CFP-10), and recombinant $M$. tuberculosis immunogenic protein MPT64 antigens were purchased from Cusabio (Selangor, Malaysia). Bovine serum albumin (BSA), human serum, gold (III) chloride trihydrate, potassium carbonate, phosphate-buffered saline (PBS) tablets, and Tween-20 were purchased from Sigma-Aldrich (St. Louis, MO, USA). 3,30,5,50-Tetramethylbenzidine (TMB) ELISA substrate and sodium citrate were obtained from Abcam (Cambridge, UK) and R\&M Chemicals (Essex, UK), respectively. Sputum samples of patients from Hospital Universiti Sains Malaysia (HUSM), Kelantan, Malaysia, were used with identification numbers TB1273/2017, B562423, TB541/2016, TB1304/2016, TB1280, and SA217. For the sputum sample, the need for consent was waived because the sputum sample is only leftover samples that have been used for laboratory purposes of HUSM. In addition, the protocol used in this study was approved by the Medical Research and Ethics Committee (MREC) of the Ministry of Health Malaysia (Approval Number: NMRR-17-3001-39473 (IIR)).

2.2. Synthesis of Gold Nanoparticles. The gold nanoparticles (AuNPs) were synthesized by citrate reduction method as previously reported [25]. A gold chloride solution was prepared by dissolving gold chloride in $100 \mathrm{ml}$ of deionized water, and the prepared solution was stored protected from light. Then, a sodium citrate solution was freshly prepared prior to use by dissolving a sodium citrate in $100 \mathrm{ml}$ of deionized water and filtered. The gold chloride solution was heated until the solution boil, and the sodium citrate was added wisely drop by drop. After $10 \mathrm{~min}$, the flask was placed into a water bath to cool down for at least $15 \mathrm{~min}$. The resulting AuNPs were then collected and stored in a refrigerator at

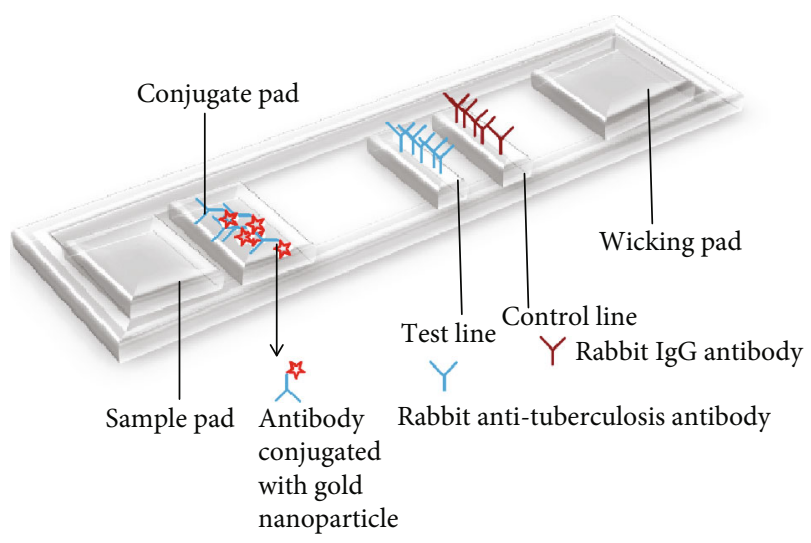

Figure 2: Design of lateral flow immunoassay (LFIA).

$4^{\circ} \mathrm{C}$ until further used. The synthesized AuNPs were then characterized using UV-Vis and HRTEM image.

2.3. Conjugation of Rabbit Anti-M. tuberculosis Antibody with AuNPs. Conjugation of rabbit anti-M. tuberculosis antibody with AuNPs was prepared according to the methods described previously [11] with slight modification. Briefly, $6 \mu \mathrm{l}$ of rabbit anti-M. tuberculosis antibody $(12 \mu \mathrm{g} / \mathrm{ml})$ was added to $5 \mathrm{ml}$ colloidal gold solution. Prior to use, the $\mathrm{pH}$ value of the prepared AuNP solution was adjusted to 8.0 by using $0.2 \mathrm{M}$ potassium carbonate and $0.1 \mathrm{M} \mathrm{HCl}$. The mixture was stirred for $10 \mathrm{~min}$, and $10 \%$ bovine serum albumin was added. The mixture was incubated for $30 \mathrm{~min}$ and stored overnight in $4^{\circ} \mathrm{C}$. After that, the mixture was centrifuged for $10 \mathrm{~min}$ at $11,000 \mathrm{~g}$ to remove unconjugated antibodies. The pellet was resuspended with passive diluent buffer (PDB) equivalent to $1 / 10$ original volume. The optical density (OD) reading was studied using UV-Vis at $540 \mathrm{~nm}$ to get $\mathrm{OD}_{540 \mathrm{~nm}}=10$. Finally, the conjugated antibody with AuNP solution was filtered through a $0.45 \mu \mathrm{m}$ cellulose acetate filter. 

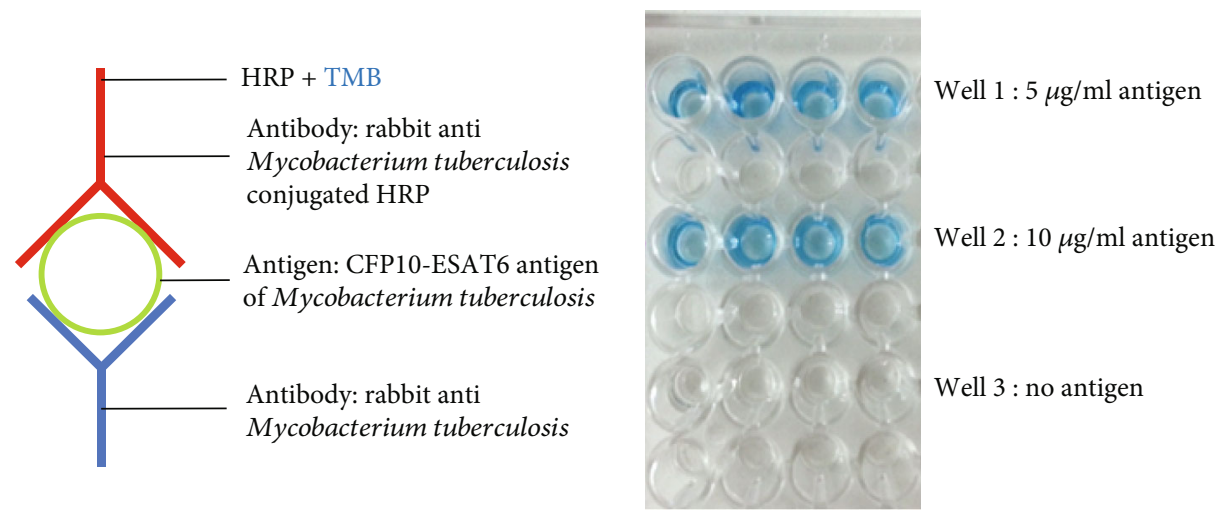

(a)
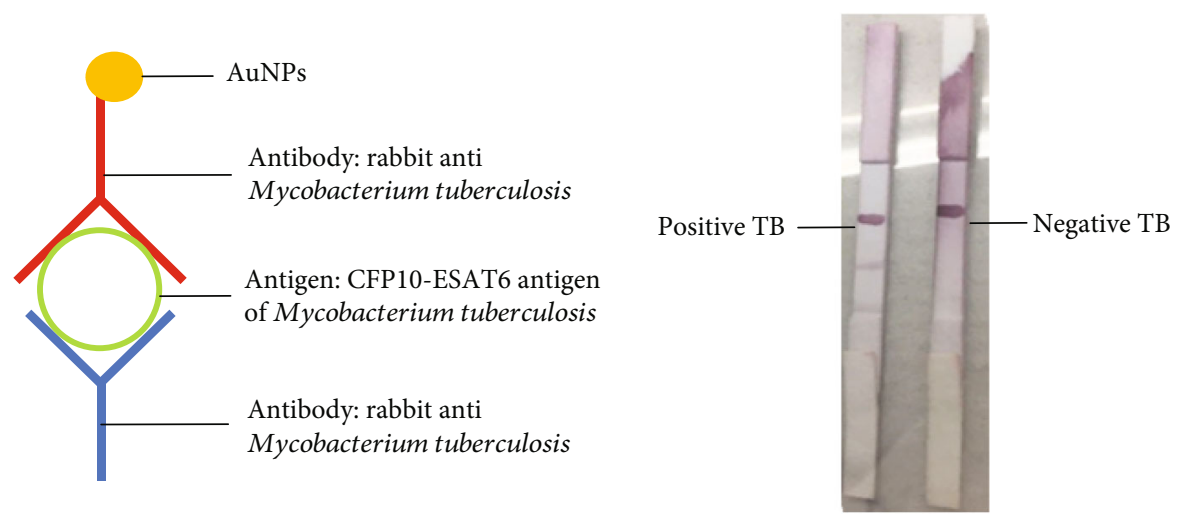

(b)

Figure 3: (a) Detection of CFP10-ESAT6 using conventional ELISA method with illustration of binding antibody-antigen complex with labelled detection antibody in sandwich form. Negative controls (without antigen) show that no colour appears in well 3, whereas wells containing antigen show blue coloured signal. (b) Detection of CFP10-ESAT6 using our developed LFIA platform with illustration of binding antibody-antigen complex in sandwich form. Two coloured lines indicate the presence of antigen, while one coloured line indicates no antigen detection.

\subsection{Spotting of Test Line and Control Line on Nitrocellulose} Membrane. Rabbit IgG antibody was dispensed on nitrocellulose membrane as control line at a concentration of $1 \mathrm{mg} / \mathrm{ml}$. Rabbit anti-M. tuberculosis antibody at a concentration of $1 \mathrm{mg} / \mathrm{ml}$ was spotted on a nitrocellulose membrane as a test line. The antibody was prepared in PBS buffer and 1\% sucrose. The nitrocellulose membrane was dried at room temperature and stored at $4^{\circ} \mathrm{C}$.

2.5. Lateral Flow Immunoassay Strips. LFIA strips were purchased from Kestrel Bio Sciences (Thailand) Co., Ltd. The LFIA construction is demonstrated in Figure 2. LFIA strip is designed with three overlapping pads placed on a backing card: sample pad (Millipore C048, $18 \times 300 \mathrm{~mm}$ ), membrane (Whatman FF 60/100, $25 \times 300 \mathrm{~mm}$ ), and wicking pad (Whatman $470,18 \times 300 \mathrm{~mm}$ ). LFIA strips were assembled on the backing card sequentially with a 1$2 \mathrm{~mm}$ overlap. The rabbit anti- $M$. tuberculosis antibody conjugated with AuNP solution with $\mathrm{OD}_{540 \mathrm{~nm}}=10$ was immobilized onto a conjugate pad $(0.5 \mu \mathrm{l}$ per $1 \mathrm{~mm})$. The conjugate pad was dried at $37^{\circ} \mathrm{C}$ for $1 \mathrm{~h}$. Then, goat antirabbit IgG antibody and rabbit anti-M. tuberculosis antibody were lined onto the nitrocellulose membrane to form the control line and the test line, respectively. The mem- branes were dried at room temperature overnight. Finally, the LFIA strips were cut into $4 \mathrm{~mm}$ wide strips and stored at $4^{\circ} \mathrm{C}$ until use.

The sputum samples were diluted with lysis buffer, and $100 \mu \mathrm{l}$ of the prepared sample was applied on the sample pad. The prepared strips were placed horizontally for 510 min to allow the sample flow from the sample pad to the wicking pad. The appearance of reddish colour at the test line and the control line was observed.

2.6. Conventional Enzyme-Linked Immunosorbent Assay (ELISA) Test. In order to confirm the positivity and negativity of the samples in comparison to our developed LFIA, a semiquantitative ELISA test was done. For this purpose, ELISA 96-well plates were coated with $100 \mu \mathrm{l}$ of rabbit antiM. tuberculosis antibody with a concentration of $1 \mu \mathrm{g} / \mathrm{ml}$ in carbonate buffer. The ELISA plate was covered with parafilm and incubated for $2 \mathrm{~h}$. Then, each coated well was washed 3 times by filling the wells with washing buffer containing PBS and Tween-20. All the solutions were removed by flicking the plate 2-3 times to remove any unbound protein. After the washing step, the ELISA plate was blocked by adding $250 \mu \mathrm{l}$ blocking buffer (PBS and BSA) for $30 \mathrm{~min}$ at room temperature. The ELISA plate was washed again 3 times by 


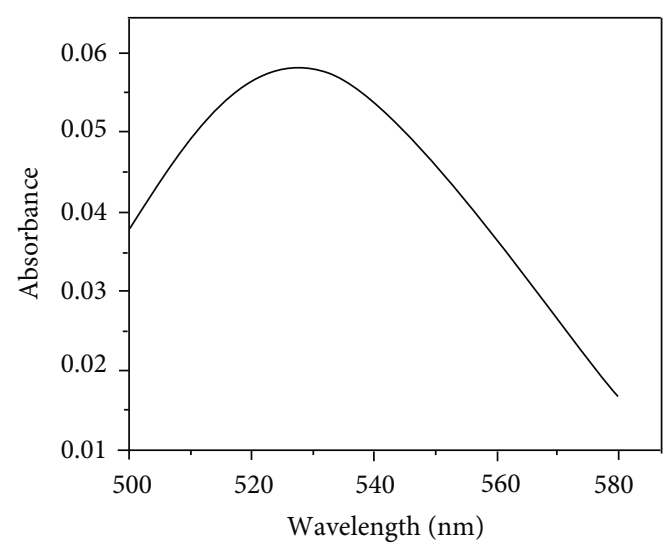

(a)

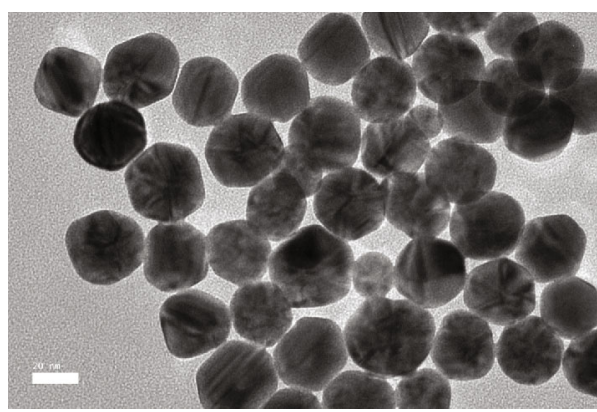

(b)

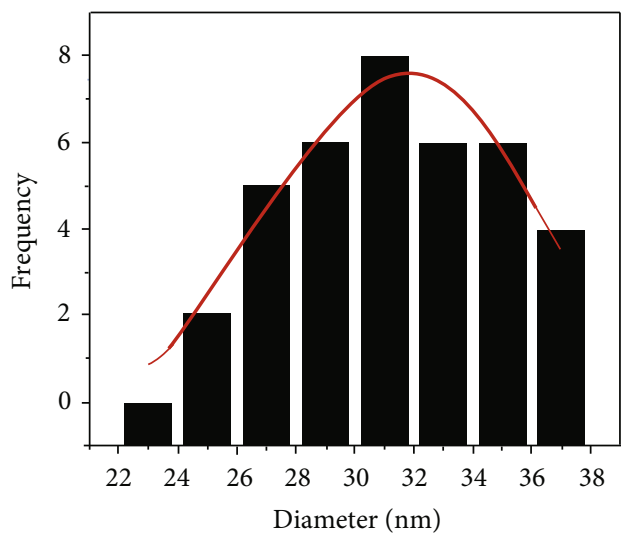

(c)

FIgure 4: Characterization of synthesized AuNPs. (a) UV-visible absorption spectrum. (b) HRTEM image of AuNPs. (c) Histogram image of nanoparticle size.

washing buffer and $100 \mu$ l of different concentrations of CFP10-ESAT6 antigen of M. tuberculosis in antibody diluent buffer containing PBS, Tween-20, and BSA were added to each well. All antigen was tested in triplicate and incubated overnight at $4^{\circ} \mathrm{C}$. After incubation of antigen, the plate was then washed again 3 times by using washing buffer and $100 \mu \mathrm{l}$ of rabbit anti-M. tuberculosis HRP conjugate was added in each well for $1 \mathrm{~h}$, followed by the washing step and addition of TMB substrate solution. Blue colour will appear which indicates the presence of labelled detection antibody with complement antibody-antigen binding. Finally, $100 \mu \mathrm{l}$ of stop solution ( $0.5 \mathrm{M} \mathrm{H}$ 2SO4) was added and the reading of absorbance was obtained by using an ELISA Microplate Reader.

\section{Results and Discussion}

3.1. Validation of Sandwich Format Using Conventional ELISA Procedure. To further explain the sensing mechanism of the sandwich strategy and evaluate the specific roles of employed antibodies, control experiments were conducted. ELISA analysis was employed to verify the binding of antibody and antigen on the test line as shown in Figure 3.

Detection antibody was labelled with HRP enzyme which will induce a coloured signal when TMB was added. The ELISA sandwich complex was formed, which includes the capture antibody, the antigen, and the detection antibody (Figure 3(a)). As can be seen in Figure 3(a), the control well shows no colour signal as the absence of antigen will cause no binding of the labelled detection antibody. The ELISA plate shows the blue signal in the wells with antigen and no blue signal can be observed in the absence of antigen. The results confirmed that the fusion protein CFP10-ESAT6 has been a great candidate antigen with high specificity for the selected antibodies used in this work based on the immunodiagnosis sandwich format in ELISA.

Even though ELISA shows an effective method for the determination of CFP10-ESAT6 in M. tuberculosis, tedious washing procedures and required antibody labelling may limit wide use of this method for simple and rapid detection of TB. Thus, in this present study, we established a simple, portable, user-friendly, and rapid platform for the determination of the anti-CFP10-ESAT6 antibody for TB diagnosis based on LFIA in sandwich assay format, as shown in Figure 3(b). The use of AuNPs enables reddish colour observation. A positive result indicates by observation both a coloured test line and a coloured control line, while an observation of a single coloured control line indicates a negative result. The current lateral flow device shows promise for use in applications where AuNPs attach through specific biological recognition events (target-binding configuration) when the target analyte (CFP10-ESAT6) is present. 


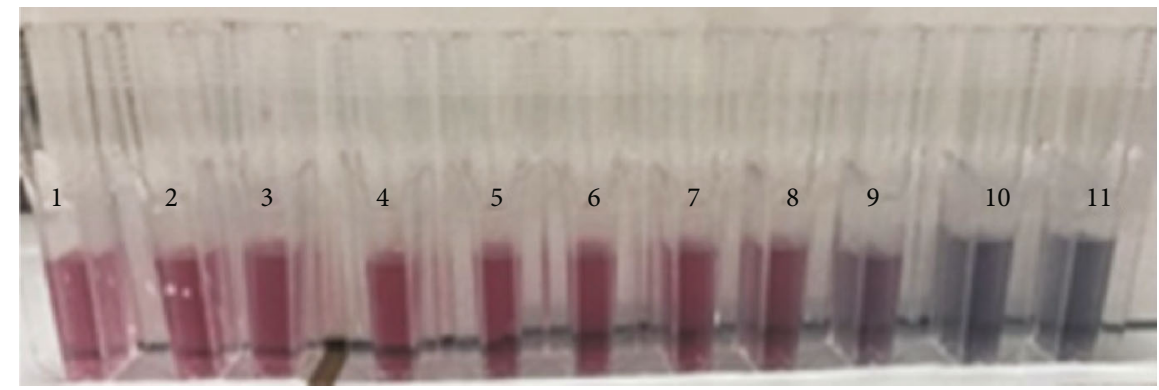

(a)

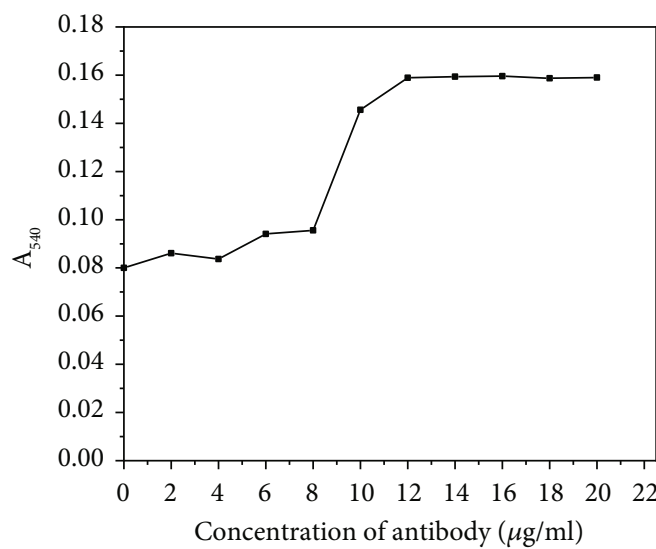

(b)

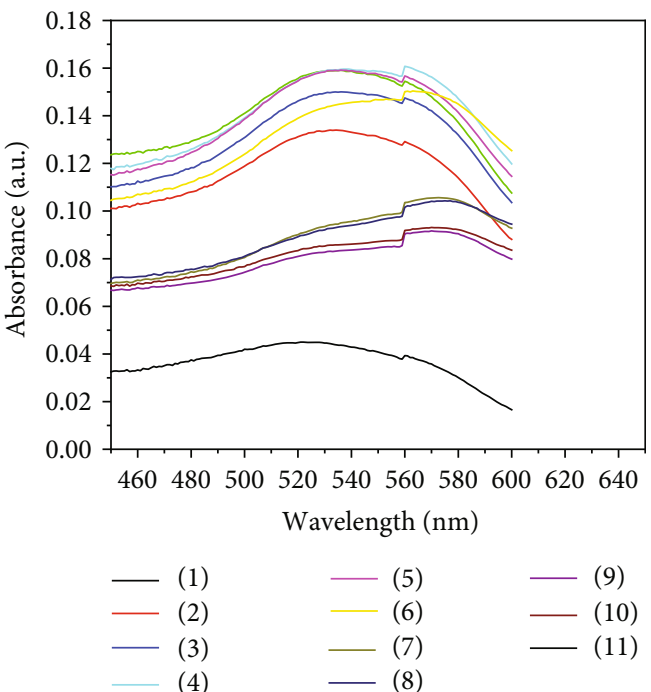

(c)

FiguRE 5: Characterization of synthesized AuNPs conjugated with antibody. (a) A series of different concentrations of antibody conjugated with AuNP solution. (b) UV-Vis peak absorption of conjugate at different rabbit anti-M. tuberculosis antibody concentrations. (c) UV-Vis peak of AuNPs incubated with various concentrations of antibodies.

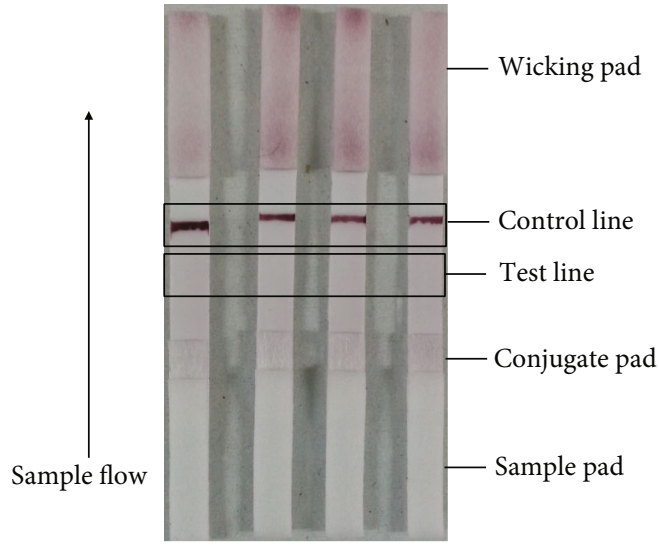

(a)

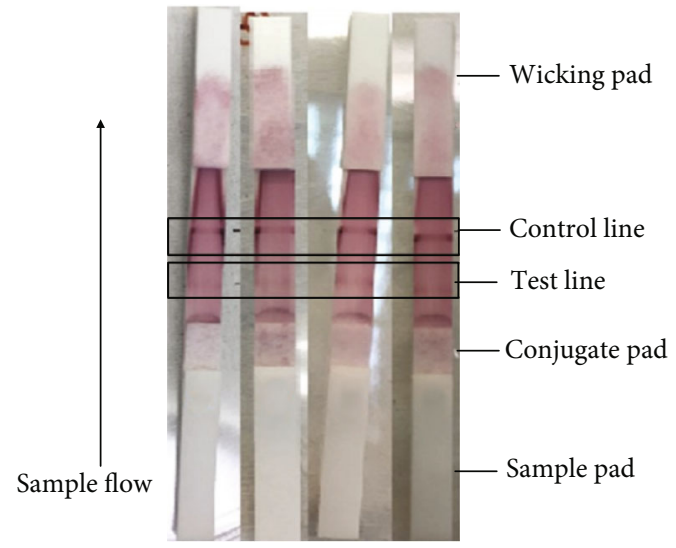

(b)

FIGURE 6: (a) The LFIA strips show reddish coloured signal only appeared at the control line when control sample (buffer solution without antigen) was loaded for 5-10 min. (b) Reddish signals presented on both test line and control line which indicate a positive signal when sputum samples of TB-positive patients 1 to 4 were dispensed on the sample pad.

3.2. Characterization of Antibody Conjugated with Gold Nanoparticles. The synthesized AuNPs were characterized by using UV-Vis and HRTEM to obtain the wavelength of the maximum absorbance $\left(\lambda_{\max }\right)$ and to determine the morphology of AuNPs which have been synthesized by citrate reduction method, respectively. Based on Figure 4(a), the 


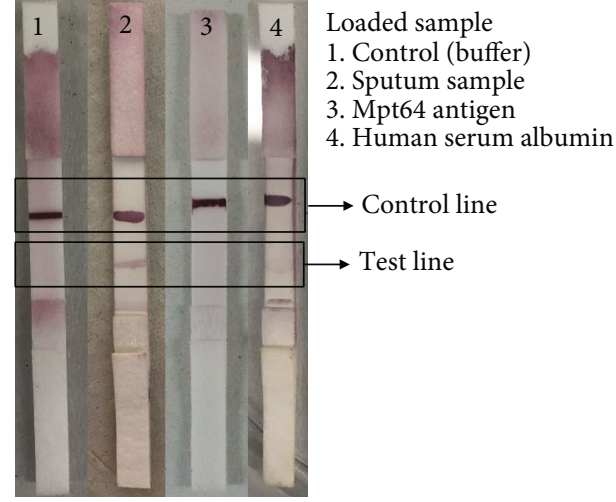

Figure 7: Specific detection of sputum sample using LFIA. The detection of CFP10-ESAT6 in sputum sample was observed as a reddish colour on the test line of the strips while no signal was detected on the test line of another target.

particle of AuNPs absorbs light at an absorbance of $520 \mathrm{~nm}$ due to the surface plasmon resonance. The HRTEM image shown in Figure 4(b) represents a clear image of spherical AuNPs less aggregated with each other, and the average size of AuNPs obtained is approximately $\sim 30 \mathrm{~nm}$ as depicted in Figure 4(c).

The AuNP labelling approach was used as a rapid detection of $M$. tuberculosis using lateral flow strips. AuNPs which were conjugated with rabbit anti-M. tuberculosis antibody were optimized to get a stable ruby-red colour for appearance as signal in the test line and the control line. The stability of the gold colloid was examined from the absorption of the colloid after addition of different concentrations of antibody. The optimal stabilizing antibody concentration was determined by using different concentrations of antibody. For conjugation, the antibodies are attached to the AuNP surface by physical and chemical interactions [11]. The antibody is nonspecifically adsorbed onto AuNPs while the stability in colloidal solution was provided by the negatively charged nanoparticles [11, 26]. The stability of the gold colloid was examined from the absorption of the colloid after addition of different concentrations of antibody.

Figure 5(a) shows the colour changes of AuNP solution containing antibody with different concentrations. The colour of the solution changed from red to blue after the addition of a low concentration of rabbit anti- $M$. tuberculosis antibody in the presence of $\mathrm{NaCl}$. The red colour of AuNPs remains at a stable state when high concentration or excess amount of antibody was added. The minimum concentration of antibody to stabilize the colloidal gold was approximately $10 \mu \mathrm{g} / \mathrm{ml}$ as shown in Figure 5(b). At $10 \mu \mathrm{g}$ of antibody per $1 \mathrm{ml}$ of gold solution, the antibody was adequately bound to AuNPs. More protein bound to the AuNPs as the concentration of antibody increase, in which all of the available binding sites were occupied. Figure 5(c) shows the UV-Vis absorbance spectra of different concentrations of antibody conjugated with AuNP solution. The maximum SPR absorption for solutions 1 to 8 (from high concentration antibodies) shows approximately at $550 \mathrm{~nm}$ while solutions 9 to 11 (lower concentration antibodies) show long wavelength shifted at $570 \mathrm{~nm}$. This is due to the aggregation of AuNPs shown in solutions 9 to 11 .

3.3. Lateral Flow Immunoassay Characterization for AntigenAntibody TB Detection. Test strips were constructed as shown in Figure 6. There are three main elements in a strip: a conjugate pad, a membrane, and an absorbent pad. Whatman membrane was used so that the flow of the sample run smoothly. The sample from the sample pad will flow through the conjugate pad until the wicking pad. The wicking pad pulled the sample over the test and control lines by trapping the excess sample. The antibody conjugated with AuNPs was deposited on the conjugate pad. The rabbit anti-M. tuberculosis was aligned on the membrane which was known to be the test line. The other line was aligned with goat antirabbit IgG antibody and known as the control line. The antibody on the test line will capture the antigen which was bound to antibody labelled with AuNPs. As a result, reddish signal colour could be observed on the test line. The rabbit IgG antibody on the control line will capture the remaining AuNP-labelled antibody and could always show signal as a validation test.

The control line on the nitrocellulose membrane was optimized so that a clear reddish signal could be observed by the naked eye. The test strips were tested by lysis buffer (no antigen), and the buffer was let to flow laterally on the nitrocellulose membrane. The reddish signal on the control line can be observed as shown in Figure 6(a) by the naked eye after 5-10 min buffer loading. Upon testing the lateral flow strips with a clinical sample of patients with positive TB, $100 \mu$ l of solution was dispensed on the sample pad. As shown in Figure 6(b), a reddish signal could be observed on the test line and the control line. This result indicates that the antibody on the test line was successfully bound with the CFP10-ESAT6 antigen in the sputum sample.

The developed LFIA was further tested with different types of antigen in order to verify the specificity of the strips. The test line of each strip which has been spot off with the antibody was observed after 5-10 min after each target flows from the sample pad to the wicking pad. As shown in Figure 7, the signal appeared on the test line of the strip when sputum sample was loaded after $10 \mathrm{~min}$. Meanwhile, no signal was observed on the test line of the control strip, which indicates the absence of any target of the sample. The results show the specific target of the antibody on the test line when no signal could be seen on the test line when the recombinant M. tuberculosis immunogenic protein MPT64 antigen and human serum albumin were tested on the strips. Therefore, the developed LFIA specifically detects the sputum sample that contains CFP10-ESAT6 and the antibody on the test line only captures the specific target of the sample.

3.4. Reproducibility Study. The reproducibility study of the developed LFIA strips was applied by preparing three strips from the same batch for each clinical sputum sample patient. Based on Figure 8, each sputum sample from different TBpositive patients (1-5) and TB-negative patient (6) was tested on LFIA strips and the results were observed after the sputum 


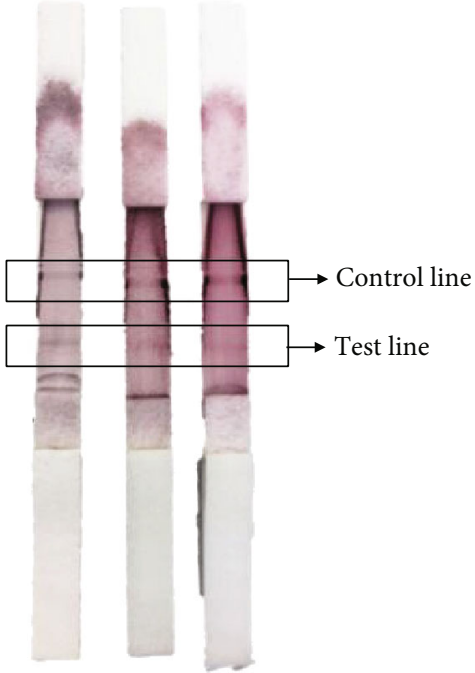

Patient 1

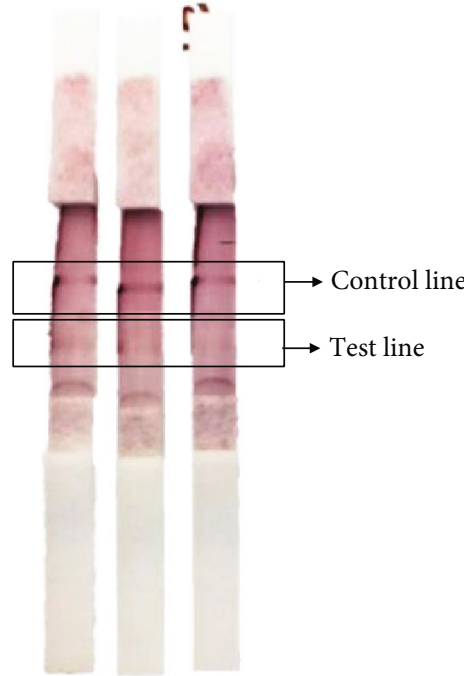

Patient 2

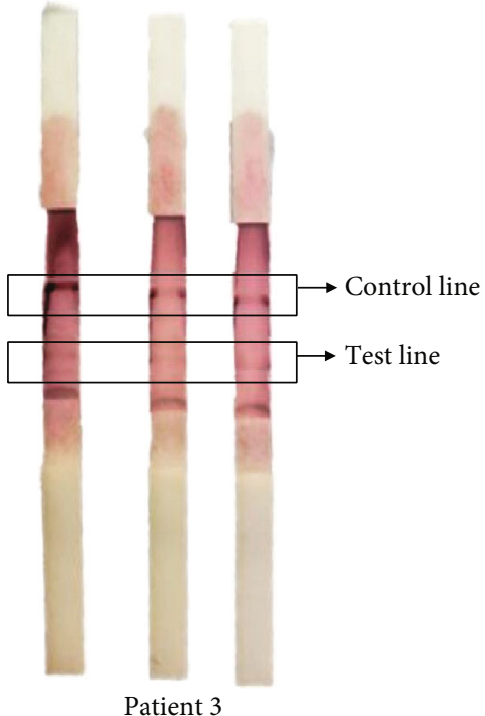

(c)

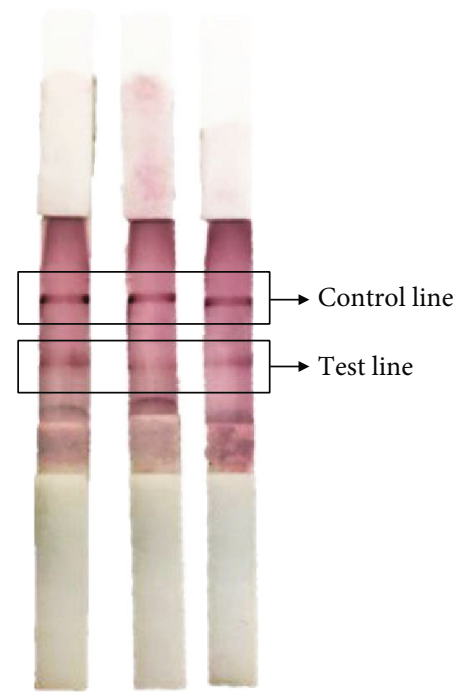

Patient 4

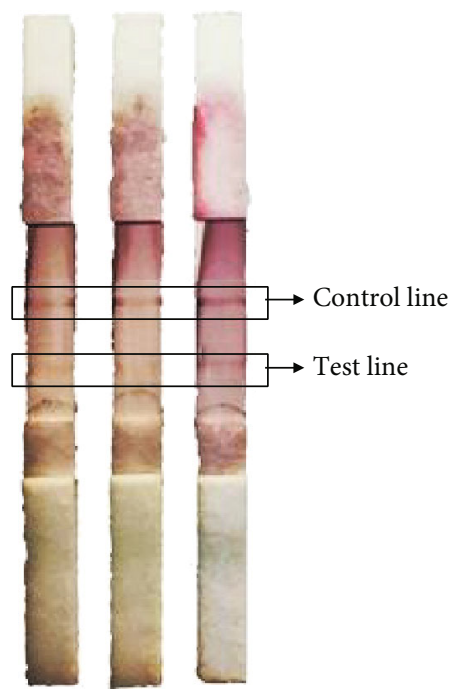

Patient 5 (b)

(e)

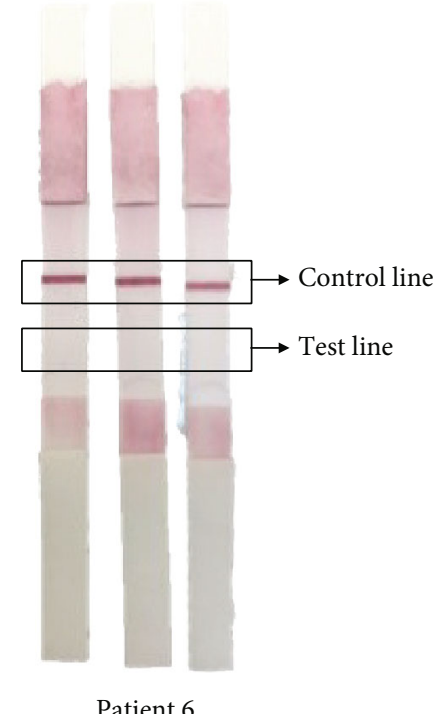

(f)

FIGURE 8: The reproducibility study of LFIA strips for detection of CFP10-ESAT6 antigen from sputum sample of TB patients. (a) Analysis for patient 1 sputum specimen, (b) analysis for patient 2 sputum specimen, (c) analysis for patient 3 sputum specimen, (d) analysis for patient 4 sputum specimen, (e) analysis for patient 5 sputum specimen, and (f) analysis for patient 6 sputum specimen.

samples were loaded on the sample pad. The results on each strip show that both the test line and the control line present signal after 5-10 min. Hence, the CFP10-ESAT6 antigen of M. tuberculosis from the clinical sputum sample of TB patients was detected by the antibody on each test line of the strips. These results suggested that the precision of each strip was assessed by randomly choosing LFIA for detection of analyte sample. To further verify that the colour change is due to the attachment of the complementary target antibody to the capture antibody on the developed LFIA strip, an additional test was employed by using the sputum sample from a TB-negative patient (patient 6). After loading the sample on the sample pad of the strip for 5-10 min, the test showed no significant colour change at the test line as com- pared to the control line, suggesting the absence of nonspecific binding of target antigen to the capture antibody on the test line zone. This proves that colour detection occurred by the attachment of complementary target antigen of $M$. tuberculosis with antibody functionalized on the developed LFIA strip.

\section{Conclusions}

We demonstrated a rapid, simple, and affordable method based on LFIA for ultrasensitive naked eye detection system of TB. The CFP10-ESAT6 detection using sandwich format procedure presented in this newly developed immunosensor shows a simple and easy platform that may provide a rapid 
alternative strategy with the potential to be used in TB diagnosis in comparison with the ELISA method. The developed sandwich immunoassay showed exceptional detection response towards CFP10-ESAT6 in sputum specimen for clinical sample application and demonstrated high sensitivity, specificity, and reproducibility as disposable-based sensor. This strip-based immunoassay could provide great potential in low-cost and POC application for TB diagnosis.

\section{Data Availability}

The data used to support the findings of this study are included within the article.

\section{Conflicts of Interest}

The authors declare that there is no conflict of interest regarding the publication of this paper.

\section{Acknowledgments}

The authors would like to thank the Ministry of Higher Education Malaysia and Universiti Putra Malaysia for the financial support through the Malaysia Research University Network (MRUN)-Universiti Putra Malaysia (UPM) (UPM/800-4/11/MRUN/2018/5539230). Special thanks also go to Siti Suraiya from HUSM Kubang Kerian, Kelantan, for helping in real sample testing.

\section{References}

[1] M. Sypabekova, A. Bekmurzayeva, R. Wang, Y. Li, C. Nogues, and D. Kanayeva, "Selection, characterization, and application of DNA aptamers for detection of Mycobacterium tuberculosis secreted protein MPT64," Tuberculosis, vol. 104, pp. 70-78, 2017.

[2] S. R. Torati, V. Reddy, S. S. Yoon, and C. Kim, "Electrochemical biosensor for Mycobacterium tuberculosis DNA detection based on gold nanotubes array electrode platform," Biosensors \& Bioelectronics, vol. 78, pp. 483-488, 2016.

[3] R. Nurmalasari, S. G. Yohan, and Y. W. Hartati, "Label-Free Electrochemical DNA Biosensor for the Detection of Mycobacterium Tuberculosis Using Gold Electrode Modified by SelfAssembled Monolayer of Thiol," Procedia Chemistry, vol. 17, pp. 111-117, 2015.

[4] J. Kim, J. Lee, K.-I. Lee, T. J. Park, H.-J. Kim, and J. Lee, "Rapid monitoring of CFP-10 during culture of Mycobacterium tuberculosis by using a magnetophoretic immunoassay," Sensors and Actuators B: Chemical, vol. 177, pp. 327-333, 2013.

[5] J.-R. Sun, S.-Y. Lee, C.-L. Perng, and J.-J. Lu, “Detecting Mycobacterium tuberculosis in Bactec MGIT 960 Cultures by Inhouse IS 6110-based PCR Assay in Routine Clinical Practice," Journal of the Formosan Medical Association, vol. 108, no. 2, pp. 119-125, 2009.

[6] M. Díaz-González, M. B. González-García, and A. CostaGarcía, "Immunosensor for Mycobacterium tuberculosis on screen-printed carbon electrodes," Biosensors and Bioelectronics, vol. 20, no. 10, pp. 2035-2043, 2005.

[7] K. L. Kaul, "Molecular detection of Mycobacterium tuberculosis: impact on patient care," Clinical Chemistry, vol. 47, no. 8, pp. 1553-1558, 2001.
[8] G. Kraus, T. Cleary, N. Miller et al., "Rapid and specific detection of the Mycobacterium tuberculosis complex using fluorogenic probes andreal-time PCR," Molecular and Cellular Probes, vol. 15, no. 6, pp. 375-383, 2001.

[9] F. Mohamad, M. M. Zaid, J. Abdullah et al., "Synthesis and characterization of polyaniline/graphene composite nanofiber and its application as an electrochemical DNA biosensor for the detection of Mycobacterium tuberculosis," Sensors, vol. 17, no. 12, p. 2789, 2017.

[10] F. Li, M. You, S. Li et al., "Paper-based point-of-care immunoassays: recent advances and emerging trends," Biotechnology Advances, p. 107442, 2019.

[11] L. A. H. van Pinxteren, P. Ravn, E. M. Agger, J. Pollock, and P. Andersen, "Diagnosis of tuberculosis based on the two specific antigens ESAT-6 and CFP10," Clinical and Vaccine Immunology, vol. 7, no. 2, pp. 155-160, 2000.

[12] G. Ruiz-Vega, M. Kitsara, M. A. Pellitero, E. Baldrich, and F. J. del Campo, "Electrochemical lateral flow devices: towards rapid immunomagnetic assays," ChemElectroChem, vol. 4, no. 4, pp. 880-889, 2017.

[13] A. Zamora-Gálvez, E. Morales-Narváez, J. Romero, and A. Merkoçi, "Photoluminescent lateral flow based on nonradiative energy transfer for protein detection in human serum," Biosensors and Bioelectronics, vol. 100, pp. 208-213, 2018.

[14] D. Y. Pereira, R. Y. T. Chiu, S. C. L. Zhang, B. M. Wu, and D. T. Kamei, "Single-step, paper-based concentration and detection of a malaria biomarker," Analytica Chimica Acta, vol. 882, pp. 83-89, 2015.

[15] D. Kim, Y. Kim, S. Hong et al., "Development of lateral flow assay based on size-controlled gold nanoparticles for detection of hepatitis B surface antigen," Sensors, vol. 16, no. 12, p. 2154, 2016.

[16] H.-K. Wang, C.-H. Tsai, K.-H. Chen et al., "Cellulose-based diagnostic devices for diagnosing serotype-2 dengue fever in human serum," Advanced Healthcare Materials, vol. 3, no. 2, pp. 187-196, 2014.

[17] J. Hu, Y.-Z. Jiang, L.-L. Wu et al., "Dual-signal readout nanospheres for rapid point-of-care detection of Ebola virus glycoprotein," Analytical Chemistry, vol. 89, no. 24, pp. 13105-13111, 2017.

[18] E. Pilavaki and A. Demosthenous, "Optimized lateral flow immunoassay reader for the detection of infectious diseases in developing countries," Sensors, vol. 17, no. 11, p. 2673, 2017.

[19] E. I. Laderman, E. Whitworth, E. Dumaual et al., "Rapid, sensitive, and specific lateral-flow immunochromatographic point-of-care device for detection of herpes simplex virus type 2-specific immunoglobulin G antibodies in serum and whole blood," Clinical and Vaccine Immunology, vol. 15, no. 1, pp. 159-163, 2008.

[20] R. Simeone, D. Bottai, W. Frigui, L. Majlessi, and R. Brosch, "ESX/type VII secretion systems of mycobacteria: insights into evolution, pathogenicity and protection," Tuberculosis, vol. 95, pp. S150-S154, 2015.

[21] M. Encinas, M. J. Marfil, S. Garbaccio et al., "Mycobacterium bovis ESAT-6, CFP-10 and EspC antigens show high conservation among field isolates," Tuberculosis, vol. 111, pp. 143-146, 2018.

[22] P. S. Renshaw, P. Panagiotidou, A. Whelan et al., "Conclusive evidence that the major T-cell antigens of the Mycobacterium tuberculosis complex ESAT-6 and CFP-10 form a tight, 1:1 
complex and characterization of the structural properties of ESAT-6, CFP-10, and the ESAT-6.CFP-10 complex: implications for pathogenesis and virulence," Journal of Biological Chemistry, vol. 277, no. 24, pp. 21598-21603, 2002.

[23] A. K. Meher, N. C. Bal, K. V. R. Chary, and A. Arora, "Mycobacterium tuberculosis H37Rv ESAT-6-CFP-10 complex formation confers thermodynamic and biochemical stability," The FEBS Journal, vol. 273, no. 7, pp. 1445-1462, 2006.

[24] P. S. Renshaw, K. L. Lightbody, V. Veverka et al., "Structure and function of the complex formed by the tuberculosis virulence factors CFP-10 and ESAT-6," The EMBO Journal, vol. 24, no. 14, pp. 2491-2498, 2005.

[25] M. Doyen, K. Bartik, and G. Bruylants, "UV-Vis and NMR study of the formation of gold nanoparticles by citrate reduction: Observation of gold-citrate aggregates," Journal of Colloid and Interface Science, vol. 399, pp. 1-5, 2013.

[26] M. H. Jazayeri, H. Amani, A. A. Pourfatollah, H. PazokiToroudi, and B. Sedighimoghaddam, "Various methods of gold nanoparticles (GNPs) conjugation to antibodies," Sensing and bio-sensing research, vol. 9, pp. 17-22, 2016. 